\title{
Cyclical Surfaces Created by Helix on General Surface of Revolution
}

\author{
Tatiana Olejníková \\ Technical University of Košice \\ Faculty of Civil Engineering, Institute of Construction Technology and Management \\ e-mail: tatiana.olejnikova@tuke.sk
}

\begin{abstract}
This paper describes the method for modelling of cyclical surfaces created by the helix on the general surface of revolution $\Phi$. The axis of the cyclical surface $\Phi_{1}$ is the helix $s$ created by screwing the point located on the surface $\Phi$ about the axis of this surface. The circle moves together with Frenet-Serret moving trihedron along the helix $s$ and creates the cyclical surface $\Phi_{1}$. The paper describes also the modelling of a rope of $n$ cyclical surfaces created by moving circles about tangent, principal normal or binormal of the helix $s$.
\end{abstract}

Key words: cyclical surface, helix, Frenet-Serret moving trihedron, transformation matrices, general surface of revolution

\section{Introduction}

Let the three-dimensional Euclidean space $\mathrm{E}^{3}$ be determined by the Cartesian coordinate system $(0, x, y, z)$. The sine curve $k$ determined by parametric equations

$$
x_{k}=a_{1}+a_{2} \sin v, y_{k}=0, z_{k}=a_{3} v, v \in\langle 0,3 \pi\rangle,
$$

where parameters $a_{1}=7, a_{2}=3, a_{3}=3$, rotates about the coordinate axis $\mathrm{z}$ and creates the surface of revolution $\Phi$ (Fig.1) determined by the vector function

$$
\mathbf{P}(u, v)=\left(x_{k} \cos u, x_{k} \sin u, z_{k}, 1\right), u \in\langle 0,2 \pi\rangle .
$$

Let the helix $s$ on the surface $\Phi$ is defined by the vector function

$$
\mathbf{r}(v)=\left(x_{s}, y_{s}, z_{s}, 1\right)=\left(x_{k} \cos m v, \operatorname{sgn} x_{k} \sin m v, z_{k}, 1\right), v \in\langle 0,3 \pi\rangle,
$$


where parameter sgn determines orientation of the helix $(\operatorname{sgn}=+1$ for right-handed and $\operatorname{sgn}=-1$ for left-handed helical movement), parameter $m$ determines the angular velocity of the helical movement of the point on the surface $\Phi$ (in Fig.2 parameters $m=3$, sgn $=+1$ ).

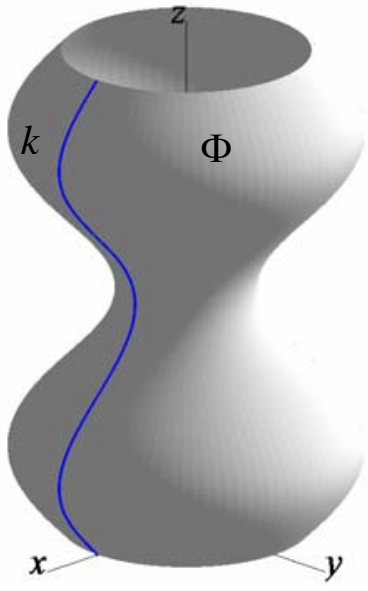

Figure 1

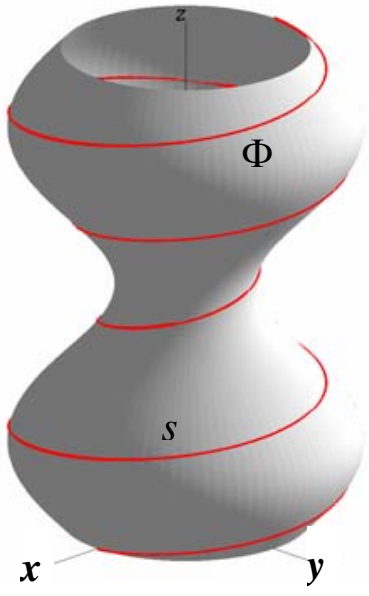

Figure 2

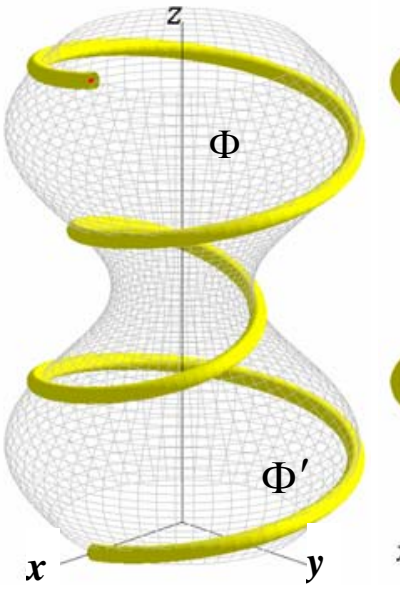

Figure 3

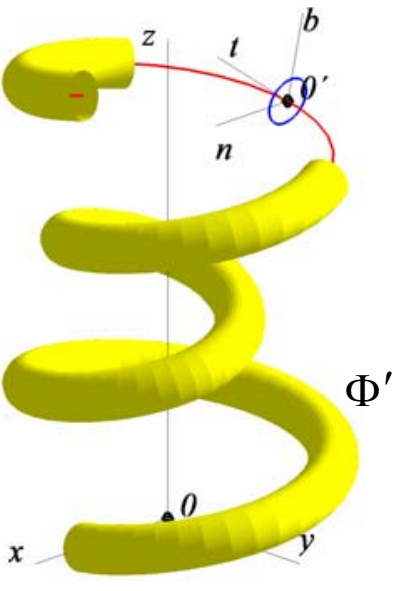

Figure 4

Let $\left(0^{\prime}, n, b, t\right)$ be the Frenet-Serret moving trihedron of the helix $s$ represented by the regular square matrix

$$
\mathbf{M}(v)=\left(\begin{array}{cccc}
n_{x}(v) & n_{y}(v) & n_{z}(v) & 0 \\
b_{x}(v) & b_{y}(v) & b_{z}(v) & 0 \\
t_{x}(v) & t_{y}(v) & t_{z}(v) & 0 \\
0 & 0 & 0 & 1
\end{array}\right),
$$

where the matrix elements are the coordinates of the unit vectors of the principle normal $n$, binormal $b$ and tangent $t$ of the helix $s$ at the point $0^{\prime} \in s$ for the coordinate system $(0, x, y, z)$

$$
\begin{gathered}
\mathbf{t}(v)=\left(t_{x}(v), t_{y}(v), t_{z}(v)\right)=\frac{\mathbf{r}^{\prime}(v)}{\left|\mathbf{r}^{\prime}(v)\right|}, \\
\mathbf{b}(v)=\left(b_{x}(v), b_{y}(v), b_{z}(v)\right)=\frac{\mathbf{r}^{\prime}(v) \times \mathbf{r}^{\prime \prime}(v)}{\left|\mathbf{r}^{\prime}(v) \times \mathbf{r}^{\prime \prime}(v)\right|}, \\
\mathbf{n}(v)=\left(n_{x}(v), n_{y}(v), n_{z}(v)\right)=\mathbf{b}(v) \times \mathbf{t}(v) .
\end{gathered}
$$

Let the moving circle $c=\left(0^{\prime}, r\right)\left(O^{\prime}\right.$ is the center, $r$ is the radius of the circle $)$ along the helix $s$ lie in the normal plane determined by the principal normal $n$ and binormal $b$ of the helix at the point $0^{\prime}$ and let it create the cyclical surface $\Phi^{\prime}$. Then the vector function of this cyclical surface $\Phi^{\prime}$ is

$$
\mathbf{P}^{\prime}(u, v)=\mathbf{r}(v)+\mathbf{c}(u) \cdot \mathbf{M}(v), u \in\langle 0,2 \pi\rangle, v \in\langle 0,3 \pi\rangle,
$$


where $\mathbf{r}(v)$ is the vector function of the helix $s$ expressed by the formula (3), $\mathbf{M}(v)$ is the transformation matrix of the coordinate system $\left(0^{\prime}, n, b, t\right)$ for the coordinate system $(0, x, y, z)$ in (4) and $\mathbf{c}(u)=(r \cos u, r \sin u, 0,1), u \in\langle 0,2 \pi\rangle$ is the vector function of the circle c. In Fig. 3 are displayed surfaces $\Phi$ and $\Phi^{\prime}$, for parameters $m=2, \operatorname{sgn}=+1$. The moving circle $c$ along the helix $s$ together with Frenet-Serret moving trihedron and cyclical surface $\Phi^{\prime}$ is displayed in Fig.4.

\section{Rope of $\boldsymbol{n}$ cyclical surfaces created by the rotation of the circles about the tangent of the helix $s$}

In this chapter we create the rope of $n$ cyclical surfaces $\Phi_{i}^{\prime \prime}, i=1, \ldots, n$ by rotation of the $n$ circles $c_{\mathrm{i}}^{\prime}=\left(O_{\mathrm{i}}^{\prime \prime}, r^{\prime}\right)$ with the center $O_{\mathrm{i}}^{\prime \prime}$ and the same radius $r^{\prime}$ about the tangent $t$ of the helix $s$. The axes of the surfaces $\Phi_{i}^{\prime \prime}$ will be helix $s_{i}^{\prime}$ created by rotation of the point with coordinates

$$
\left(x_{0 \mathrm{i}}, y_{0 \mathrm{i}}, z_{0 \mathrm{i}}, 1\right)=\left(x_{\mathrm{i}}, y_{\mathrm{i}}, z_{\mathrm{i}}, 1\right) .\left(\begin{array}{cccc}
\cos m^{\prime} v & \operatorname{sgn} \sin ^{\prime} \sin v & 0 & 0 \\
-\operatorname{sgn} \sin ^{\prime} m^{\prime} v & \cos m^{\prime} v & 0 & 0 \\
0 & 0 & 1 & 0 \\
0 & 0 & 0 & 1
\end{array}\right) \text {, }
$$

where

$$
\left(x_{\mathrm{i}}, y_{\mathrm{i}}, z_{\mathrm{i}}, 1\right)=(d \cos \alpha \mathrm{i}, d \sin \alpha \mathrm{i}, 0,1), \alpha=2 \pi / n \text {. }
$$

Parameter $d$ determines the distance between centres of the circles $d=\left|0^{\prime} O_{\mathrm{i}}^{\prime \prime}\right|$, parameter $\alpha$ is the angle $\alpha=\angle O_{i}^{\prime \prime} O^{\prime} O_{i+1}^{\prime \prime}$ (see Fig.5), parameter $m^{\prime}$ is the angular velocity of the movement and parameter sgn' determines the orientation of the movement $\left(\operatorname{sgn}^{\prime}=+1\right.$ for right-handed and $\operatorname{sgn}^{\prime}=-1$ for left-handed rotation). Then the vector function of the helix $s_{\mathrm{i}}^{\prime}$ is

$$
\begin{gathered}
\mathbf{r}_{\mathrm{i}}^{\prime}(v)=\left(x_{s_{\mathrm{i}}^{\prime}}, y_{s_{\mathrm{i}}}, z_{s_{\mathrm{i}}^{\prime}}, 1\right)=\mathbf{r}(v)+\left(x_{0 \mathrm{i}}, y_{0 \mathrm{i}}, z_{0 \mathrm{i}}, 1\right) \cdot \mathbf{M}(v), \\
x_{s_{\mathrm{i}}^{\prime}}=x_{s}+x_{0 \mathrm{i}} \cdot n_{x}(v)+y_{0 \mathrm{i}} \cdot b_{x}(v)+z_{0 \mathrm{i}} \cdot t_{x}(v), \\
y_{s_{\mathrm{i}}^{\prime}}=y_{s}+x_{0 \mathrm{i}} \cdot n_{y}(v)+y_{0 \mathrm{i}} \cdot b_{y}(v)+z_{0 \mathrm{i}} \cdot t_{y}(v), \\
z_{s_{\mathrm{i}}^{\prime}}=z_{s}+x_{0 \mathrm{i}} \cdot n_{z}(v)+y_{0 \mathrm{i}} \cdot b_{z}(v)+z_{0 \mathrm{i}} \cdot t_{z}(v) .
\end{gathered}
$$

Let $\left(0^{\prime \prime}, n_{\mathrm{i}}^{\prime}, b_{\mathrm{i}}^{\prime}, t_{\mathrm{i}}^{\prime}\right)$ be the Frenet-Serret moving trihedrons of the helix $s_{\mathrm{i}}^{\prime}$ represented by regular square matrices

$$
\mathbf{M}_{\mathrm{i}}^{\prime}(v)=\left(\begin{array}{cccc}
n_{x \mathrm{i}}^{\prime}(v) & n_{y \mathrm{i}}^{\prime}(v) & n_{z \mathrm{i}}^{\prime}(v) & 0 \\
b_{x \mathrm{i}}^{\prime}(v) & b_{y \mathrm{i}}^{\prime}(v) & b_{z \mathrm{i}}^{\prime}(v) & 0 \\
t_{x \mathrm{i}}^{\prime}(v) & t_{y \mathrm{i}}^{\prime}(v) & t_{\mathrm{zi}}^{\prime}(v) & 0 \\
0 & 0 & 0 & 1
\end{array}\right)
$$


where the matrix elements are the coordinates of the unit vectors of the principle normals $n_{\mathrm{i}}^{\prime}$, binormals $b_{\mathrm{i}}^{\prime}$ and tangents $t_{\mathrm{i}}^{\prime}$ of the helix $s_{\mathrm{i}}^{\prime}$ at the points $O_{\mathrm{i}}^{\prime \prime} \in s_{\mathrm{i}}^{\prime}$ in the coordinate system $(0, x, y, z)$

$$
\begin{gathered}
\mathbf{t}_{\mathbf{i}}^{\prime}(v)=\left(t_{x \mathrm{i}}^{\prime}(v), t_{y \mathrm{i}}^{\prime}(v), t_{z \mathrm{i}}^{\prime}(v)\right)=\frac{\mathbf{r}_{\mathbf{i}}^{\prime}(v)^{\prime}}{\left|\mathbf{r}_{\mathbf{i}}^{\prime}(v)^{\prime}\right|}, \\
\mathbf{b}_{\mathbf{i}}^{\prime}(v)=\left(b_{x \mathrm{i}}^{\prime}(v), b_{y \mathrm{i}}^{\prime}(v), b_{z \mathrm{i}}^{\prime}(v)\right)=\frac{\mathbf{r}_{\mathbf{i}}^{\prime}(v)^{\prime} \times \mathbf{r}_{\mathbf{i}}^{\prime}(v)^{\prime \prime}}{\left|\mathbf{r}_{\mathbf{i}}^{\prime}(v)^{\prime} \times \mathbf{r}_{\mathbf{i}}^{\prime}(v)^{\prime \prime}\right|}, \\
\mathbf{n}_{\mathbf{i}}^{\prime}(v)=\left(n_{x \mathrm{i}}^{\prime}(v), n_{y \mathrm{i}}^{\prime}(v), n_{z \mathrm{i}}^{\prime}(v)\right)=\mathbf{b}_{\mathbf{i}}^{\prime}(v) \times \mathbf{t}_{\mathbf{i}}^{\prime}(v) .
\end{gathered}
$$

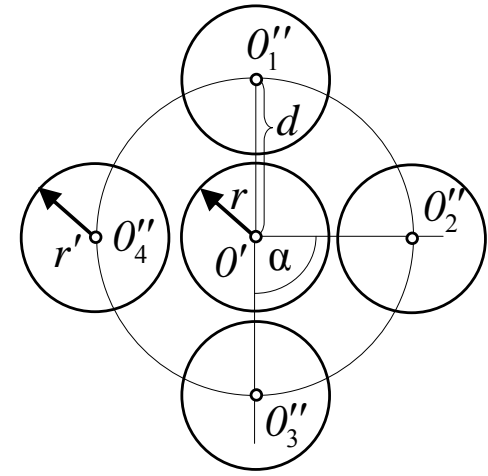

Figure 5

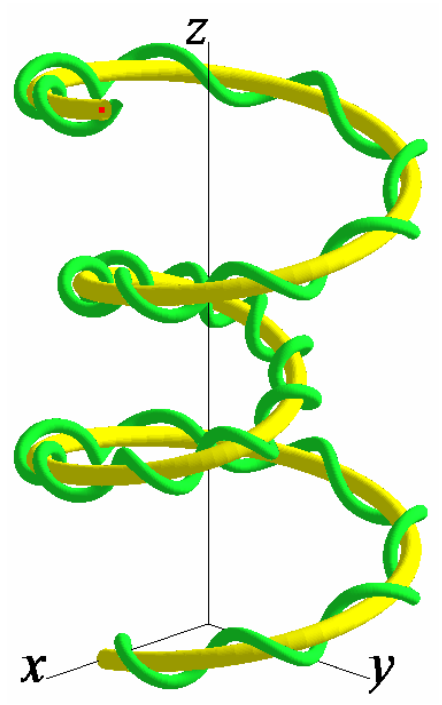

Figure 7

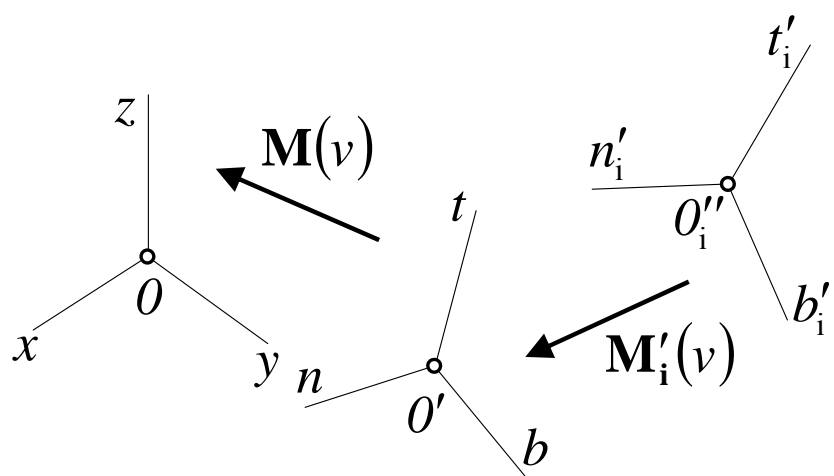

Figure 6

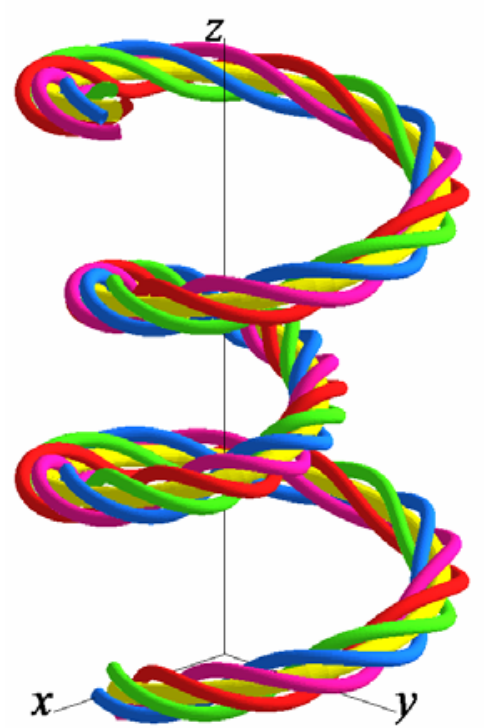

Figure 8 


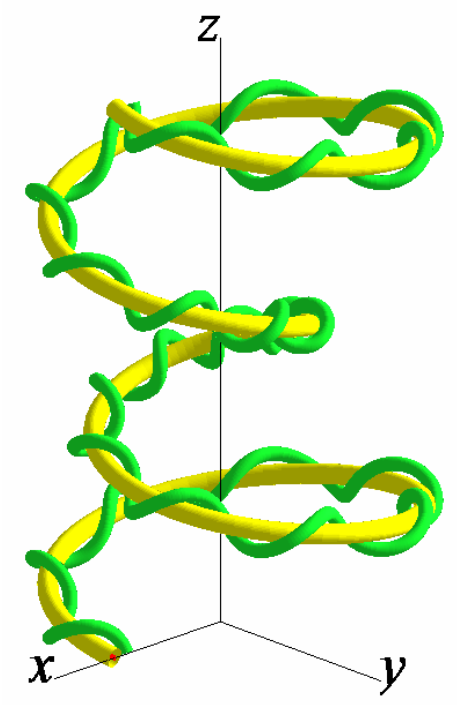

Figure 9

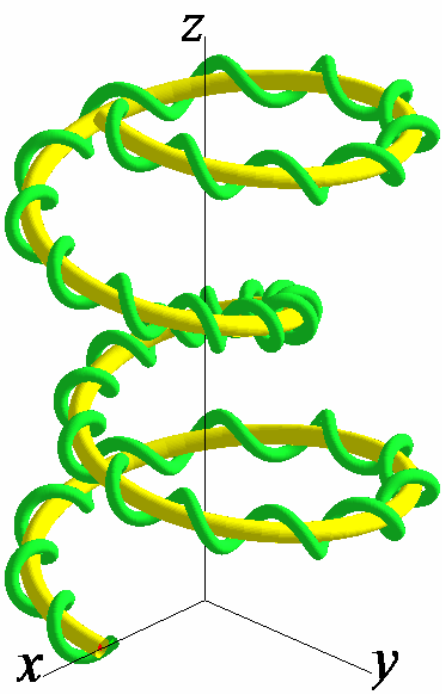

Figure 10

Let the moving circles $c_{\mathrm{i}}^{\prime}=\left(O_{\mathrm{i}}^{\prime \prime}, r^{\prime}\right)$ along the helix $s_{\mathrm{i}}^{\prime}$ lie in the normal planes determined by the principal normals $n_{\mathrm{i}}^{\prime}$ and binormals $b_{\mathrm{i}}^{\prime}$ of the helix at the points $0_{\mathrm{i}}^{\prime \prime} \in s_{\mathrm{i}}^{\prime}$ and let them create the cyclical surfaces $\Phi_{\mathrm{i}}^{\prime \prime}$. Then the vector functions of these cyclical surfaces $\Phi_{\mathrm{i}}^{\prime \prime}$ are

$$
\mathbf{P}_{\mathbf{i}}^{\prime \prime}(u, v)=\mathbf{r}_{\mathbf{i}}^{\prime}(v)+c_{\mathrm{i}}^{\prime}(u) . \mathbf{M}_{\mathbf{i}}^{\prime}(v), u \in\langle 0,2 \pi\rangle, v \in\langle 0,3 \pi\rangle
$$

where $\mathbf{r}_{\mathbf{i}}^{\prime}(v)$ are vector functions of the helix $s_{i}^{\prime}$ expressed by the equation $(11), \mathbf{M}_{\mathbf{i}}^{\prime}(v)$ is the transformation matrix of the coordinate systems $\left(0_{i}^{\prime \prime}, n_{i}^{\prime}, b_{i}^{\prime}, t_{i}^{\prime}\right)$ into the coordinate system $(0, x, y, z)$ in (15) and $c_{\mathrm{i}}^{\prime}(u)$ are the vector functions of the circles determined by their centers $O_{i}^{\prime \prime}$ and radius $r^{\prime}$.

In Fig. 6 there are displayed transformations of the coordinate system $\left(0^{\prime}, n, b, t\right)$ (moving trihedron of the helix $s$ ) into the coordinate system $(0, x, y, z)$ and the transformation of the coordinate system $\left(0^{\prime \prime}, n_{\mathrm{i}}^{\prime}, b_{\mathrm{i}}^{\prime}, t_{\mathrm{i}}^{\prime}\right)$ (moving trihedrons of the helix $s_{\mathrm{i}}^{\prime}$ ) into the coordinate system $\left(0^{\prime}, n, b, t\right)$.

In Fig.7 are displayed left-handed cyclical surfaces, the surface $\Phi^{\prime}$ for $n=1$ and $\Phi_{1}^{\prime \prime}$, determined by parameters $m^{\prime}=8$, sgn' $=-1$ and in In Fig. 8 for $n=4$ right-handed cyclical surfaces $\Phi_{i}^{\prime \prime}, i=1, . ., 4$ determined by parameters $m^{\prime}=12$, sgn' $=+1$.

In Figs.9, 10 are displayed surfaces $\Phi^{\prime}$ for parameters $m=2$, sgn $=-1$ and $n=1$ left-handed surface $\Phi_{i}^{\prime \prime}$ determined for parameters $m^{\prime}=8$, sgn' $=-1$ or the right-handed surface $\Phi_{i}^{\prime \prime}$ for parameters $m^{\prime}=12, \operatorname{sgn}^{\prime}=+1$. 


\section{Rope of $\boldsymbol{n}$ cyclical surfaces created by the rotation of the circle about the principle normal of the helix $s$}

In this chapter we create the rope of $n$ cyclical surfaces $\Phi_{i}^{\prime \prime}, i=1, \ldots, n$ by the revolution of the $n$ circles $c_{\mathrm{i}}^{\prime}=\left(O_{\mathrm{i}}^{\prime \prime}, r^{\prime}\right)\left(O_{\mathrm{i}}^{\prime \prime}\right.$ are centres and $r^{\prime}$ is the same radius of circles) around the principal normal $n$ of the helix s. Let the axes of the surfaces $\Phi_{\mathrm{i}}^{\prime \prime}$ is the helix $s_{\mathrm{i}}^{\prime}$ created by rotating of the point for coordinates

$$
\left(x_{0 \mathrm{i}}, y_{0 \mathrm{i}}, z_{0 \mathrm{i}}, 1\right)=\left(x_{\mathrm{i}}, y_{\mathrm{i}}, z_{\mathrm{i}}, 1\right) \cdot\left(\begin{array}{cccc}
1 & 0 & 0 & 0 \\
0 & \cos m^{\prime} v & \operatorname{sgn}^{\prime} \sin m^{\prime} v & 0 \\
0 & -\operatorname{sgn}^{\prime} \sin m^{\prime} v & \cos m^{\prime} v & 0 \\
0 & 0 & 0 & 1
\end{array}\right),
$$

where

$$
\left(x_{\mathrm{i}}, y_{\mathrm{i}}, z_{\mathrm{i}}, 1\right)=(0,-d \sin \alpha \mathrm{i}, d \cos \alpha \mathrm{i}, 1), \alpha=2 \pi / n \text {. }
$$

Parameter $d$ determines the distance between centres of the circles $c$ and $c_{\mathrm{i}}^{\prime}, d=\left|0^{\prime} O_{\mathrm{i}}^{\prime \prime}\right|$, parameter $\alpha$ is the angle $\alpha=\angle O_{i}^{\prime \prime} O^{\prime} O_{i+1}^{\prime \prime}$ (see Fig.5), parameter $m^{\prime}$ is the angular velocity of the movement and parameter sgn' determines the orientation of the movement $\left(\operatorname{sgn}^{\prime}=+1\right.$ for right-handed and $\mathrm{sgn}^{\prime}=-1$ for left-handed rotation). Then the vector function of the helix $s_{\mathrm{i}}^{\prime}$ is

$$
\mathbf{r}_{\mathbf{i}}^{\prime}(v)=\left(x_{s_{\mathrm{i}}^{\prime}}, y_{s_{\mathrm{i}}^{\prime}}, z_{s_{\mathrm{i}}^{\prime}}, 1\right)=\mathbf{r}(v)+\left(x_{0 \mathrm{i}}, y_{0 \mathrm{i}}, z_{0 \mathrm{i}}, 1\right) \cdot \mathbf{M}(v),
$$

Let the moving circles $c_{\mathrm{i}}^{\prime}=\left(O_{\mathrm{i}}^{\prime \prime}, r^{\prime}\right)$ around the helix $s_{\mathrm{i}}^{\prime}$ lie in the normal planes determined by principal normals $n_{\mathrm{i}}^{\prime}$ and binormals $b_{\mathrm{i}}^{\prime}$ of the helix at the points $O_{\mathrm{i}}^{\prime \prime} \in s_{\mathrm{i}}^{\prime}$ and let them create the cyclical surfaces $\Phi_{\mathrm{i}}^{\prime \prime}$. Then the vector functions of such cyclical surfaces $\Phi_{\mathrm{i}}^{\prime \prime}$ are

$$
\mathbf{P}_{\mathbf{i}}^{\prime \prime}(u, v)=\mathbf{r}_{\mathbf{i}}^{\prime}(v)+c_{\mathrm{i}}^{\prime}(u) \cdot \mathbf{M}_{\mathbf{i}}^{\prime}(v), u \in\langle 0,2 \pi\rangle, v \in\langle 0,3 \pi\rangle
$$

where $\mathbf{r}_{\mathbf{i}}^{\prime}(v)$ are vector functions of the helix $s_{i}^{\prime}$ expressed by the equation (11), $\mathbf{M}_{\mathbf{i}}^{\prime}(v)$ is the transformation matrix of the coordinate systems $\left(O_{\mathrm{i}}^{\prime \prime}, n_{\mathrm{i}}^{\prime}, b_{\mathrm{i}}^{\prime}, t_{\mathrm{i}}^{\prime}\right)$ into the coordinate system $(0, x, y, z)$ in (15) and $c_{\mathrm{i}}^{\prime}(u)$ are vector functions of the circles determined by their centers $0_{\mathrm{i}}^{\prime \prime}$ and radius $r^{\prime}$.

In Fig.10 there are displayed the helix $s(m=2$, sgn $=+1)$ together with $n=1$ the righthanded surface $\Phi_{1}^{\prime \prime}$ determined by parameters $m^{\prime}=10$, sgn' ${ }^{\prime}=+1$, in Fig.11 with $n=1$ the left-handed surface $\Phi_{1}^{\prime \prime}$ determined by parameters $m^{\prime}=12$, sgn' $=-1$ and in Fig. 12 are $n=4$ left-handed cyclical surfaces $\Phi_{\mathrm{i}}^{\prime \prime}, \mathrm{i}=1, . ., 4$ determined by parameters $m^{\prime}=8, \operatorname{sgn}^{\prime}=-1$. 


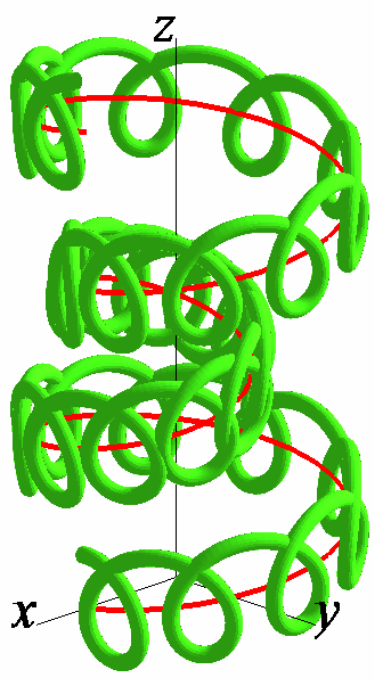

Figure 10

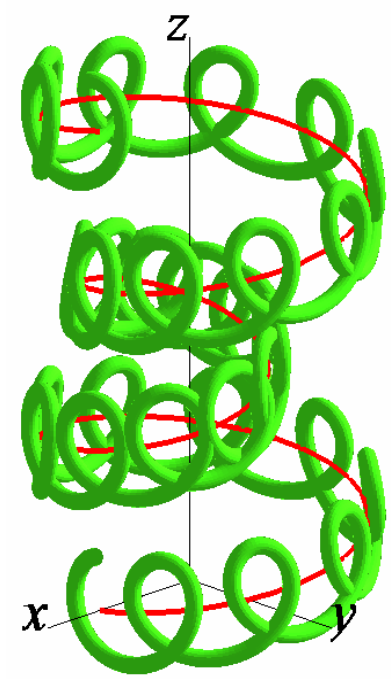

Figure 11

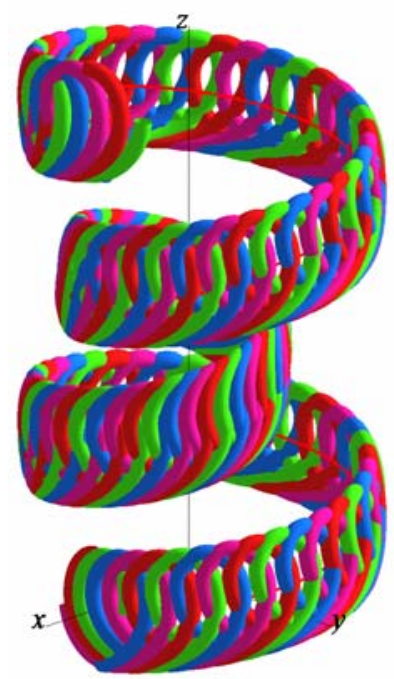

Figure 12

\section{Rope of $\boldsymbol{n}$ cyclical surfaces created by the rotation of the circle about the binormal of the helix $s$}

In this chapter we create a rope of $n$ cyclical surfaces $\Phi_{i}^{\prime \prime}, \mathrm{i}=1, \ldots, n$ by revolution of the $n$ circles $c_{\mathrm{i}}^{\prime}=\left(O_{\mathrm{i}}^{\prime \prime}, r^{\prime}\right)$ about the binormal $b$ of the helix $s$. Axes of the surfaces $\Phi_{\mathrm{i}}^{\prime \prime}$ will be helix $s_{\mathrm{i}}^{\prime}$ created by revolving of the point with coordinates

$$
\left(x_{0 \mathrm{i}}, y_{0 \mathrm{i}}, z_{0 \mathrm{i}}, 1\right)=\left(x_{\mathrm{i}}, y_{\mathrm{i}}, z_{\mathrm{i}}, 1\right) .\left(\begin{array}{cccc}
\cos m^{\prime} v & 0 & \operatorname{sgn}^{\prime} \sin m^{\prime} v & 0 \\
0 & 1 & 0 & 0 \\
0 & -\operatorname{sgn}^{\prime} \sin m^{\prime} v & \cos m^{\prime} v & 0 \\
0 & 0 & 0 & 1
\end{array}\right) \text {, }
$$

where

$$
\left(x_{\mathrm{i}}, y_{\mathrm{i}}, z_{\mathrm{i}}, 1\right)=(d \cos \alpha \mathrm{i}, 0, d \sin \alpha \mathrm{i}, 1), \alpha=2 \pi / n \text {. }
$$

The vector function of the helix $s_{\mathrm{i}}^{\prime}$ is

$$
\mathbf{r}_{\mathbf{i}}^{\prime}(v)=\left(x_{s_{\mathrm{i}}^{\prime}}, y_{s_{\mathrm{i}}^{\prime}}, z_{s_{\mathrm{i}}^{\prime}}, 1\right)=\mathbf{r}(v)+\left(x_{0 \mathrm{i}}, y_{0 \mathrm{i}}, z_{0 \mathrm{i}}, 1\right) \cdot \mathbf{M}(v),
$$

and vector functions of the cyclical surfaces $\Phi_{\mathrm{i}}^{\prime \prime}$ are the same as in the previous chapter

$$
\mathbf{P}_{\mathbf{i}}^{\prime \prime}(u, v)=\mathbf{r}_{\mathbf{i}}^{\prime}(v)+c_{\mathrm{i}}^{\prime}(u) . \mathbf{M}_{\mathbf{i}}^{\prime}(v), u \in\langle 0,2 \pi\rangle, v \in\langle 0,3 \pi\rangle \text {. }
$$




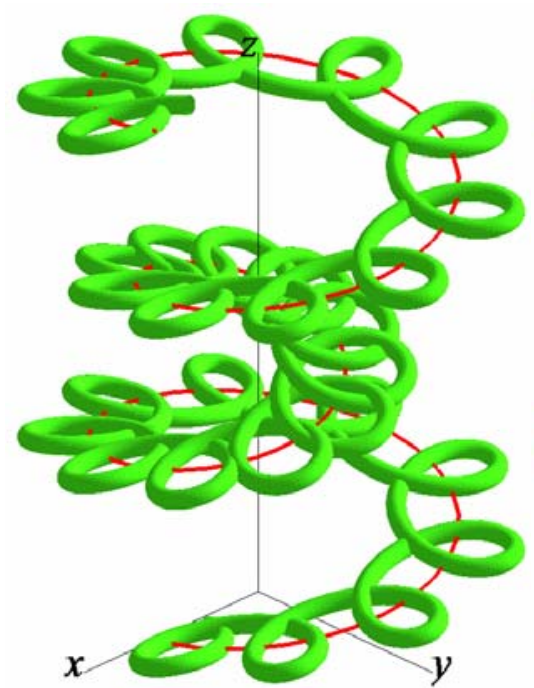

Figure 13

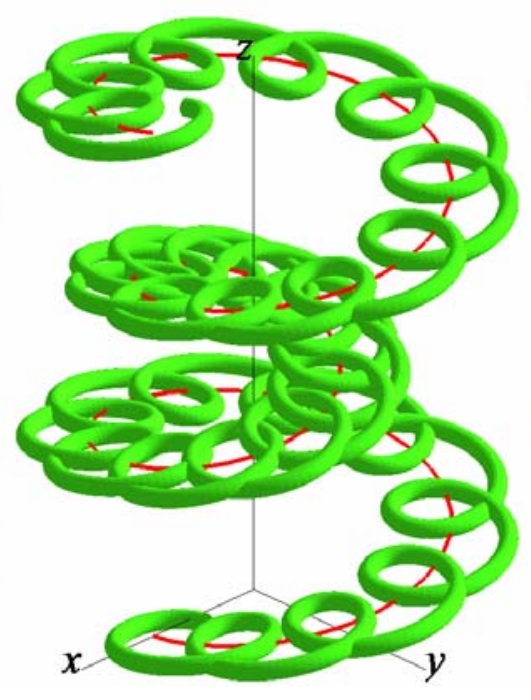

Figure 14

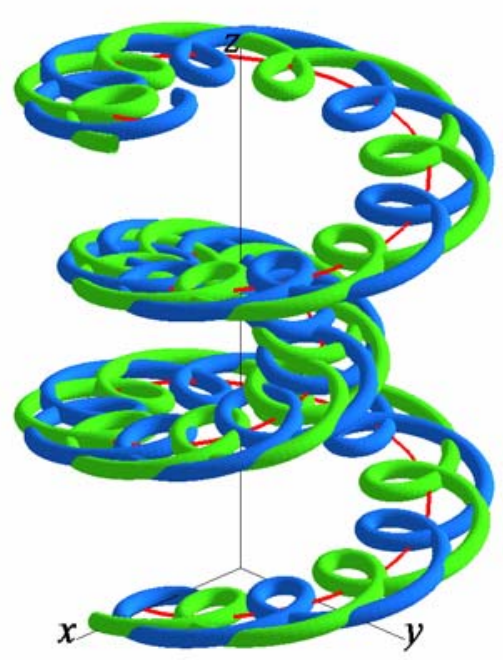

Figure 15

In Fig.13 there are displayed helix $s(m=2, \operatorname{sgn}=+1)$ together with $n=1$ right-handed cyclical surface $\Phi_{1}^{\prime \prime}$ with parameters $m^{\prime}=10, \operatorname{sgn}^{\prime}=+1$, in Fig.14 with $n=1$ left-handed surface $\Phi_{1}^{\prime \prime}$ with parameters $m^{\prime}=10$, sgn' $=-1$ and in Fig.15 $n=2$ left-handed cyclical surfaces $\Phi_{\mathrm{i}}^{\prime \prime}$ with parameters $m^{\prime}=6$, $\operatorname{sgn}^{\prime}=-1$.

\section{Conclusion}

At the conclusion, it can be summarised that presented family of cyclical surfaces serves as an endlessly rich source of inspiration for artistic and design purposes. Their unusually complex forms obtained in a relatively simple way of composite spatial transformation.

\section{Acknowledgements}

This work was supported by VEGA 1/0321/12 „Theoretical and experimental analysis of adaptive cable and tensegrity systems under static and dynamic stress considering the effect of wind and seismic".

\section{References}

[1] Budinský, B. \& Kepr, B. (1970) Basic of Differetial Geometry with Technical Applications. Praha, SNTL - Publishers of Technical Literature

[2] Olejníková, T. (2009). Two-Revolutional Cyclical Surface. Acta Polytechnica Hungarica, Journal of Applied Sciences. Vol.6, Issue 4, pp. 101-110

[3] Olejníková, T. (2013). Helical one, two, three-revolutional Cyclical surfaces. Global Journal of Science Frontier research F, Mathematics and Decision Sciences. Vol.13, Issue 4, pp.46-56 